\title{
Two characteristic temperatures for a Bose-Einstein condensate of a finite number of particles
}

\author{
Z. Idziaszek ${ }^{1,2}$ and K. Rzążewski ${ }^{1,3}$ \\ ${ }^{1}$ Centrum Fizyki Teoretycznej, Polska Akademia Nauk, 02-668 Warsaw, Poland \\ ${ }^{2}$ Institut für Theoretische Physik, Universität Hannover, D-30167 Hannover, Germany \\ ${ }^{3}$ Dept. of Physics and Astronomy, University of Rochester, NY 14627, USA
}

\begin{abstract}
We consider two characteristic temperatures for a Bose-Einstein condensate, that are related to certain properties of the condensate statistics. We calculate them for an ideal gas confined in power-law traps and show that they approach the critical temperature in the limit of large number of particles. The considered characteristic temperatures can be useful in the studies of Bose-Einstein condensates of a finite number of atoms, indicating the point of a phase transition.
\end{abstract}

PACS numbers: 03.75.Hh, 05.30.Jp

Experimental achievement of Bose-Einstein condensation (BEC) in trapped, cold gases of alkali atoms [1], has stimulated large interest in the physics of this phenomenon. Among others, the issue of fluctuations in the number of condensed atoms has been a subject of intensive theoretical studies, both for noninteracting

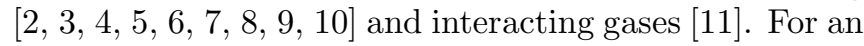
ideal Bose gas, described in the canonical or microcanonical ensemble, the condensate fluctuations are maximal just below the critical temperature. The point of maximal fluctuations defines some characteristic temperature, which can indicate the occurence of a phase-transition [9]. In the systems containing finite number of particles, the thermodynamic functions remain analytic for all temperatures, and definition of the critical temperature cannot be based on the presence of a singularity in the thermodynamic functions. In this case, the characteristic temperature provides an alternative way to localize the point of a phase-transition.

Different characteristic temperature can be defined on the basis of the ground-state occupation number. Mean ground-state population as a function of the temperature, exhibits the presence of the inflexion point close to the critical temperature. In this paper we study the properties of both characteristic temperatures, demonstrating that they tend to the critical temperature in the limit of large number of particles.

The definition of a suitable characteristic temperature for finite systems was already considered by several authors. One of the candidates was a cross-over temperature. It is the temperature at which the probability distribution of the occupation of the condensed state changes its character from the one with the maximum at zero atoms (above phase transition) to the one with the maximum at nonzero (below the phase transition) 7, 8, 12, 13. Another candidate was the maximum in the specific heat [14, 15, 16]. We stress that there are other characteristic temperatures possible, all tending to the critical one in the limit of $N$ going to infinity and, perhaps, one of them may be less difficult to measure in the experiment or compute rigorously for the interacting system.
We describe the system of $N$ noninteracting bosons, at a temperature $T$, using the canonical ensemble. The canonical partition function $Z(N, T)$, is, by definition related to grand canonical one $\Xi(z, T)$, through equation: $\Xi(z, T)=\sum_{N=0}^{\infty} z^{N} Z(N, T)$. The grand canonical partition function is known in the compact form: $\Xi(z, T)=\prod_{\nu=0}^{\infty}\left(1-z e^{-\beta \varepsilon_{\nu}}\right)^{-1}$ where $\beta=1 /\left(k_{B} T\right)$, and $\varepsilon_{\nu}$ is the single-particle energy of the level $\nu$. Unfortunately, no such general expression exists for $Z(N, T)$, and we calculate $Z(N, T)$ from $\Xi(z, T)$, using Cauchy integral formula

$$
Z(N, T)=\oint \frac{d z}{2 \pi i} \frac{\Xi(z, T)}{z^{N+1}} .
$$

In the similar manner, it is possible to express the ground-state occupation number $\left\langle N_{0}\right\rangle$ in the canonical ensemble

$$
\left\langle N_{0}\right\rangle=\frac{1}{Z(N, T)} \oint \frac{d z}{2 \pi i} \frac{\Xi(z, T)}{z^{N+1}} \frac{z}{1-z},
$$

and its fluctuations $\left\langle\delta^{2} N_{0}\right\rangle$

$$
\begin{aligned}
\left\langle\delta^{2} N_{0}\right\rangle & =\left\langle N_{0}^{2}-N_{0}\right\rangle+\left\langle N_{0}\right\rangle-\left\langle N_{0}\right\rangle^{2}, \\
\left\langle N_{0}^{2}-N_{0}\right\rangle & =\frac{2}{Z(N, T)} \oint \frac{d z}{2 \pi i} \frac{\Xi(z, T)}{z^{N+1}}\left(\frac{z}{1-z}\right)^{2} .
\end{aligned}
$$

Standard way of calculating the contour integrals (1), (2) and (4) is to use the saddle point (SP) approximation. This method exploits the fact that for large number of atoms the integrand is sharply peaked around the most probable value, and can be approximated by the Gaussian function. This approximation, however, ceases to be valid below the critical temperature, $T_{C}$, of the BEC, when the saddle point lies close to the ground-state singularity. In this case SP method predicts improper results for the condensate fluctuations at low temperatures 4, 5, 10] Alternatively, the Maxwell Demon (MD) ensemble [6] can be used, in which the moments of the condensate statistics can be easily elaborated from the grand canonical partition function of excited subsystem. This approach, however, is not suitable for studying the 
condensate statistics in the transition region, and in the case of fluctuations, it does not predict the occurence of the maximum 6, 7, 8].

In this paper we apply a variant of the SP method, developed quite recently by Holthaus and Kalinovski [10]. Following the idea of Dingle [17], the authors of [10] exclude the ground-state term from the Taylor expansion around the SP and perform further calculation with the special treatment of the ground-state term. In this way, they calculate mean number of condensed atoms and its fluctuations, in the whole range of temperatures, including the most interesting regime of the phase transition. According to [10] we rewrite Eq. (11) in the form

$$
Z(N, T)=\oint \frac{d z}{2 \pi i} \frac{\exp (-F(z, T))}{1-z},
$$

where $F(z, T)=(N+1) \ln z+\sum_{\nu=1}^{\infty} \ln \left(1-z e^{-\beta \varepsilon_{\nu}}\right)$ is a tempered function, with excluded contribution of the ground-state term. Function $F(z, T)$ should be expanded around the saddle point $z_{0}$, which is calculated for the whole integrand. It fulfills the following equation

$$
\frac{z_{0}}{1-z_{0}}+\sum_{\nu=1}^{\infty} \frac{z_{0} e^{-\beta \varepsilon_{\nu}}}{1-z_{0} e^{-\beta \varepsilon_{\nu}}}=N+1 .
$$

Expansion of $F(z, T)$ around $z_{0}$ up to the second-order terms, leads to an integral involving a product of the Gaussian function with the singular ground-state term $1 /(1-z)$. The result of integration may be expressed in terms of the parabolic cylinder function [10]. In the regime of condensation, it can be approximated by relatively simple expression

$$
Z(N, \beta)=e^{-F\left(z_{0}, T\right)-1}
$$

The same procedure may be repeated for the contour integrals (2) and (4), describing $\left\langle N_{0}\right\rangle$ and $\left\langle\delta^{2} N_{0}\right\rangle$, respectively. The SP of the former integral, $z_{1}$, fulfills the following equation

$$
2 \frac{z_{1}}{1-z_{1}}+\sum_{\nu=1}^{\infty} \frac{z_{1} e^{-\beta \varepsilon_{\nu}}}{1-z_{1} e^{-\beta \varepsilon_{\nu}}}=N
$$

while the SP of the latter integral, $z_{1}$, has to be determined from

$$
3 \frac{z_{2}}{1-z_{2}}+\sum_{\nu=1}^{\infty} \frac{z_{2} e^{-\beta \varepsilon_{\nu}}}{1-z_{2} e^{-\beta \varepsilon_{\nu}}}=N-1 .
$$

Similar procedure, as that used for derivation of (7), applied to the integrals (2) and (4), yields the following results 10

$$
\begin{aligned}
\left\langle N_{0}\right\rangle & =2 \frac{z_{1}}{1-z_{1}} e^{F\left(z_{0}, T\right)-F\left(z_{1}, T\right)-1} \\
\left\langle N_{0}^{2}-N_{0}\right\rangle & =9\left(\frac{z_{2}}{1-z_{2}}\right)^{2} e^{F\left(z_{0}, T\right)-F\left(z_{2}, T\right)-2}
\end{aligned}
$$

Eqs. (10)-(11), with the saddle points $z_{0}, z_{1}$ and $z_{2}$ calculated from Eqs. (6), (8), and (9) respectively, determine the mean ground-state occupation number $\left\langle N_{0}\right\rangle$ and the fluctuations $\left\langle\delta^{2} N_{0}\right\rangle$ for temperatures $T<T_{C}$. Comparison with the exact numerical results calculated for moderate-size systems containing $10^{2}-10^{4}$ atoms demonstrate, that the modified SP method predicts very accurate values of $\left\langle N_{0}\right\rangle$ and $\left\langle\delta^{2} N_{0}\right\rangle$. The accuracy of the SP approximation grows with the number of atoms, which reflects the fact, that for larger $N$ the integrand becomes more sharply peaked in the SP.

Derivation of the analytical results for the considered characteristic temperatures, requires to solve, at least in some approximation, the implicit equations determining the saddle points. The approximate solutions of Eqs. (6), (8) and (9) may be found, by expanding their left hand side around $z=1$, without altering the groundstate term $z /(1-z)$. Applying this procedure to Eq. (6), we obtain

$$
\frac{z_{0}}{1-z_{0}}+n_{e}(T)+d_{e}(T)\left(z_{0}-1\right) \simeq N+1
$$

where

$$
n_{e}(T)=\sum_{\nu=0}^{\infty} \frac{e^{-\beta \varepsilon_{\nu}}}{1-e^{-\beta \varepsilon_{\nu}}}
$$

and

$$
d_{e}(T)=\sum_{\nu=0}^{\infty} \frac{e^{-\beta \varepsilon_{\nu}}}{1-e^{-\beta \varepsilon_{\nu}}}\left(\frac{e^{-\beta \varepsilon_{\nu}}}{1-e^{-\beta \varepsilon_{\nu}}}+1\right)
$$

After expansion, the SP Eq. (12) becomes quadratic in $z_{0}$. Its solution reads

$$
z_{0}=\frac{n_{0}+2 d_{e}-\sqrt{n_{0}^{2}+4 d_{e}}}{2 d_{e}}
$$

where $n_{0}(T)=N-n_{e}(T)+2$. The same procedure repeated for the two remaining SP Eqs. (8) and (9), leads to the following solutions

$$
\begin{aligned}
& z_{1}=\frac{n_{0}+2 d_{e}-\sqrt{n_{0}^{2}+8 d_{e}}}{2 d_{e}}, \\
& z_{2}=\frac{n_{0}+2 d_{e}-\sqrt{n_{0}^{2}+12 d_{e}}}{2 d_{e}} .
\end{aligned}
$$

The distance of the saddle points $z_{1}$ and $z_{2}$ to $z_{0}$, in the complex plane, is very small, which may be easily seen from Eqs. (15), (16), (17). Hence, $F\left(z_{1}, T\right)$ and $F\left(z_{2}, T\right)$, appearing in Eqs. (10) and (11), may be calculated by means of the Taylor expansion around $z=z_{0}$. This will allow to further simplify Eqs. (10) and (11). In order to preserve sufficient accuracy required for calculation of the characteristic temperature, we perform expansion up to the second order terms in the small parameters $\left(z_{1}-z_{0}\right)$ and $\left(z_{2}-z_{0}\right)$ for $F\left(z_{1}, T\right)$ and $F\left(z_{2}, T\right)$, respectively. In 


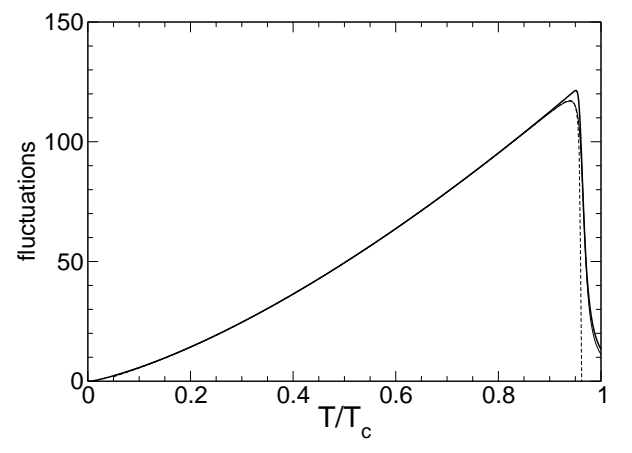

FIG. 1: Canonical fluctuations of the condensate occupation number for $N=10^{4}$ atoms. Thick solid line depicts the exact numerical result. Thin solid line corresponds to the numerical result calculated from Eqs. (10) and (11) derived in the SP approximation. Dashed lines represents the analytical result (22).

the case of $F\left(z_{1}, T\right)$, we obtain

$$
\begin{aligned}
F\left(z_{1}, T\right) & \simeq F\left(z_{0}, T\right)+\frac{z_{1}-z_{0}}{1-z_{0}}- \\
& -\frac{1}{2}\left(d_{e}(T)+\frac{z_{0}}{1-z_{0}}\right)\left(\frac{z_{2}-z_{0}}{z_{0}}\right)^{2},
\end{aligned}
$$

where we have applied the following approximation $\frac{\partial^{2} F}{\partial z^{2}}\left(z_{0}\right) \simeq-d_{e}(T)-z_{0} /\left(1-z_{0}\right)$. The small error that results from this approximation is, in fact, a higher order term, which may be safely neglected. In the same way we expand $F\left(z_{2}, T\right)$, and substitute the expanded functions into (10) and (11), obtaining

$$
\begin{aligned}
\left\langle N_{0}\right\rangle & =2 \frac{z_{1}}{1-z_{1}} \times \\
& \times e^{\left(\frac{z_{0}-z_{1}}{z_{0}}\right)^{2}\left[\frac{z_{0}}{1-z_{0}}\left(\frac{1}{2}+\frac{z_{0}}{z_{0}-z_{1}}\right)+\frac{1}{2} d_{e}(T)\right]-1}, \\
\left\langle N_{0}^{2}-N_{0}\right\rangle & =9\left(\frac{z_{2}}{1-z_{2}}\right)^{2} \times \\
& \times e^{\left(\frac{z_{0}-z_{2}}{z_{0}}\right)^{2}\left[\frac{z_{0}}{1-z_{0}}\left(\frac{1}{2}+\frac{z_{0}}{z_{0}-z_{2}}\right)+\frac{1}{2} d_{e}(T)\right]-2} .
\end{aligned}
$$

We have performed several numerical tests, which proved that Eqs. (19)-(20), with the saddle points given by (15)(17), predict very accurate values of $\left\langle N_{0}\right\rangle$ and $\left\langle\delta^{2} N_{0}\right\rangle$. In comparison to the initial Eqs. (10) and (11), they do not require numerical solving of equations for the saddle points. Nevertheless, developed set of equations is still too complicated, for the analytical calculation of the characteristic temperature. To this end, we expand Eqs. (15)-(17) and (19)-(20) in the power series in small parameter $N^{-1}$, assuming that $n_{e} \sim N$ and $d_{e} \sim N$. After straightforward, but tedious calculations we arrive at

$$
\begin{aligned}
\left\langle N_{0}\right\rangle & =n_{0}+\frac{1}{2} \frac{d_{e}}{n_{0}}-\frac{3}{2} \\
\left\langle\delta^{2} N_{0}\right\rangle & =d_{e}-\frac{3}{2}\left(\frac{d_{e}}{n_{0}}\right)^{2}-\frac{3}{2} \frac{d_{e}}{n_{0}}-2,
\end{aligned}
$$

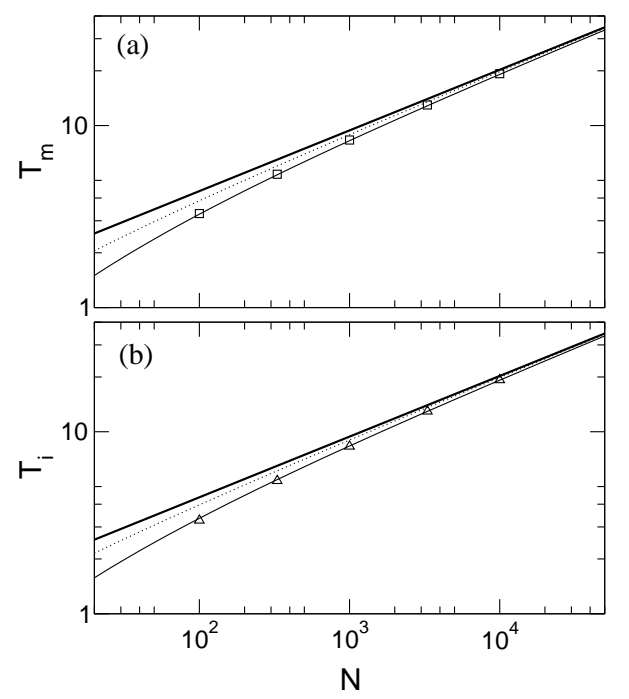

FIG. 2: Temperature of the maximal fluctuations $T_{m}$ (a) and the temperature of the inflexion point $T_{i}$ (b) as a function of the number of atoms $N$, calculated in the canonical ensemble for the case of 3D harmonic trap. Thick solid line on both plots depicts the critical temperature calculated in thermodynamic limit. Figure (a): $T_{m}$ given by Eq. (27) (dotted line), is compared with the analytical result accounting also for lower order terms (thin solid line), and with exact data of numerical calculations (squares). Figure (b): $T_{i}$ given by Eq. (28) (dotted line) is compared with the analytical result accounting also for lower order terms (thin solid line), and with exact data of numerical calculations (triangles).

where in the final result we include the terms of the order of $O(1)$, and higher. In the thermodynamic limit, only the leading order terms are important: $n_{0}$ in Eq. (21) and $d_{e}$ in Eq. (22). Those terms can be derived from the MD ensemble [6]. The inclusion of the remaining terms is, however, crucial for the occurrence of the inflexion point in $\left\langle N_{0}\right\rangle$, and the maximum in $\left\langle\delta^{2} N_{0}\right\rangle$.

Figure 1 shows the fluctuations $\left\langle\delta^{2} N_{0}\right\rangle$ for an ideal gas of $N=10^{4}$ atoms, confined in 3D harmonic trap. The exact numerical result calculated by means of recurrence relations, is compared with predictions of Eqs. (11) and (22). In the case of Eq. (11), the saddle points $z_{0}, z_{1}$, and $z_{2}$, are calculated numerically from Eqs. (6), (8), and (9), respectively. It is clearly seen that Eq. (111) obtained in the SP approximation, fits very well the exact numerical curve, apart from the region close to the maximum, where a small discrepancy is present. This difference, however, does not influence significantly the temperature of the maximal fluctuations. On the other hand, in a wide range of temperatures Eqs.(11) and (22) gives almost identical values of $\left\langle\delta^{2} N_{0}\right\rangle$. Their predictions are different in the vicinity of the critical temperature, where $n_{0} \simeq 0$ and Eq. (22) becomes invalid. It should be stressed, however, that this wrong behavior at $T_{C}$ does not influence the position of maximum, which remains exactly the same as in (11).

Now, we turn to the calculation of the temperature 
of maximal fluctuations $T_{m}$ and the temperature of inflexion point $T_{i}$. We consider an ideal Bose gas confined in $D$-dimensional trap with power-law single-particle energy spectra. The condensate fluctuations in such systems have been studied [7, 8]; in this paper we adopt the notation of [8]. The energy spectra is given by $\varepsilon_{\left\{\nu_{i}\right\}}=\Delta \sum_{i=1}^{D} \nu_{i}^{\sigma}$, where $\Delta$ is the energy gap between the ground-state and the first excited state, $\sigma$ is an exponent depending on the shape of potential, and $\nu_{i}$ are the integer numbers counting the excitations in $i$-th cartesian direction. Here, we assume the case of spherically symmetric potential, stressing that our analysis can be extended for general case of an anisotropic trap. Moreover, we are interested in the case $D>\sigma$, in which the condensation occurs below the critical temperature $T_{C}$ given by $k_{B} T_{C} / \Delta=(N / \zeta(d))^{1 / d} \Gamma(1+1 / \sigma)^{-\sigma}$ [7, 8], where $d=D / \sigma$, and $\zeta(d)$ denotes Riemann's Zeta function. In power-law traps, functions $n_{e}(T)$ and $d_{e}(T)$ are given by [7, 8]

$$
\begin{gathered}
n_{e}(T)=N\left(\frac{T}{T_{c}}\right)^{d}, \\
d_{e}(T)=\alpha N\left(\frac{T}{T_{c}}\right)^{\gamma},
\end{gathered}
$$

where we retain only the leading order terms. In Eq. (24), the dimensionless coefficients $\gamma$ and $\alpha$ are given by $\alpha=\zeta(d-1) / \zeta(d), \gamma=d$ in the case of $d>2$, while for $1<d<2$ they are given by $\alpha=\Gamma(1+1 / \sigma)^{D-2 \sigma} \zeta(3-$ d) $\Gamma(d)^{-1} \zeta(d)^{-2 / d}, \gamma=2$. In the border case: $d=2$, $d_{e}(T)$ exhibits logarithmic dependence on $T$, however, we will not consider this special case. We substitute Eqs. (23) and (24) into Eqs. (21) and (22), and determine the characteristic temperatures from: $\left.\frac{d\left\langle\delta^{2} N_{0}\right\rangle}{d T}\right|_{T=T_{m}}=0$ and $\left.\frac{d^{2}\left\langle N_{0}\right\rangle}{d T^{2}}\right|_{T=T_{i}}=0$, obtaining

$$
\begin{aligned}
\frac{T_{m}}{T_{c}} & =1-\frac{(3 \alpha)^{1 / 3}}{d} N^{\frac{(\gamma / d)-2}{3}}, \\
\frac{T_{i}}{T_{c}} & =1-\frac{1}{d}\left(\frac{\alpha d}{d-1}\right)^{1 / 3} N^{\frac{(\gamma / d)-2}{3}} .
\end{aligned}
$$

It is worth stressing that the power-law depence on $N$ is exactly the same for $T_{m}$ and $T_{i}$. In the considered regime of parameters $\gamma / d<2$, and both characteristic temperatures approach $T_{C}$ when $N \rightarrow \infty$. In the specific case, of 3D harmonic potential $(d=3, \gamma=3)$, Eqs. (25) and (26) take form

$$
\begin{aligned}
\frac{T_{m}}{T_{c}} & =1-\left(\frac{\zeta(2)}{9 \zeta(3)}\right)^{1 / 3} N^{-1 / 3} \\
\frac{T_{i}}{T_{c}} & =1-\left(\frac{\zeta(2)}{18 \zeta(3)}\right)^{1 / 3} N^{-1 / 3} .
\end{aligned}
$$

Figure 2 shows the dependence of $T_{m}$ (upper plot) and $T_{i}$ (lower plot) on the number of atoms $N$, in the case of $3 \mathrm{D}$ harmonic trap. It compares the predictions of Eqs. (25) and (26) with exact results of numerical calculations. In addition it also presents the analytical result, derived with the inclusion of the lower order terms in $n_{e}(T)$ and $d_{e}(T)$. In this case we obtain the same $N^{-1 / 3}$ dependence of $T_{m}$ and $T_{i}$ as in Eqs. (27) and (28), but with a different prefactors equal to $\zeta(2) / 2 \zeta(3)^{2 / 3}+(\zeta(2) / 9 \zeta(3))^{1 / 3}$ and $\zeta(2) / 2 \zeta(3)^{2 / 3}+(\zeta(2) / 18 \zeta(3))^{1 / 3}$, respectively. From Fig. 2 we see that Eqs. (27) and (28) predicts slightly different values in comparison to the exact data. Nevertheless, the analytical curve accountinng also for lower order terms fits very well the numerical results.

In conclusion, we have studied two characteristic temperatures for a Bose-Einstein condensate, defined by the point of maximal fluctuations in the ground-state occupation number and by the inflexion point of the groundstate occupation number. For a wide class of power-law traps, we have calculated both characteristic temperatures, showing that they approach the critical temperature in the limit of large number of particles. The numerical calculations performed for 3D harmonic trap, reveal a good agreement with our analytical results. The concept of the characteristic temperature may be useful in the studies of the BEC with a finite number of atoms, where the characteristic temperature can indicate the occurence of a phase transition.

Z.I. acknowledges support from Polish KBN Grant No. 5-P03B-103-20 and the Alexander von Humboldt Stiftung.
[1] M.H. Anderson et al., Science 269, 198 (1995); K.B. Davis et al., Phys. Rev. Lett. 75, 3969 (1995); C.C. Bradley et al., ibid 75, 1687 (1995); 79, 1170 (1997).

[2] H.D. Politzer, Phys. Rev. A 54, 5048 (1996).

[3] M. Wilkens and C. Weiss, J. Mod. Opt. 44, 1801 (1997).

[4] M. Gajda and K. Rzążewski, Phys. Rev. Lett. 78, 2686 (1997).

[5] S. Grossmann and M. Holthaus, Phys. Rev. Lett. 79, 3557 (1997).

[6] P. Navez et al., Phys. Rev. Lett. 79, 1789 (1997);

[7] S. Grossmann and M. Holthaus, Opt. Ex. 1, 262 (1997).
[8] C. Weiss and M. Wilkens, Opt. Ex. 1, 272 (1997).

[9] H.-J. Schmidt and J. Schnack, Physica A 260, 479 (1998).

[10] M. Holthaus and E. Kalinowski, Ann. Phys. (N. Y.) 10, 385 (1999).

[11] S. Giorgini, L. P. Pitaevskii, and S. Stringari, Phys. Rev. Lett. 80, 5040 (1998); Z. Idziaszek et al., Phys. Rev. Lett. 82, 4376 (1999); V.V. Kocharovsky, Vl.V. Kocharovsky, and M.O. Scully, Phys. Rev. Lett. 84, 2306 (2000); H. Xiong et al., Phys. Rev. A 65, 033609 (2002).

[12] V.V. Kocharovsky et al., Phys. Rev. A 61023609 (2000). 
[13] M. Wilkens et al., J. Phys. B 33, 779 (2000).

[14] K. Kirsten and D.J. Toms, Phys. Rev. A 54, 4188 (1996).

[15] H. Haugerud, T. Haugest, and F. Ravndal, Phys. Lett. A 225, 18 (1997).

[16] R.K. Pathria, Phys. Rev. A 58, 1490 (1998).
[17] R.B. Dingle, Asymptotic Expansions: Their Derivations and Interpretation (Academic Press, London and New York, 1973). 